\title{
The impact of supplementation with pomegranate fruit (Punica granatum L.) juice on selected antioxidant parameters and markers of iron metabolism in rowers
}

\author{
A. Urbaniak' ${ }^{1}$ P. Basta ${ }^{2}$, K. Ast $^{1}$, A. Wołoszyn ${ }^{3}$, J. Kuriańska - Wołoszyn ${ }^{3}$, Ewa Latour $^{4}$ \\ and A. Skarpańska - Stejnborn ${ }^{1 *}$
}

\begin{abstract}
Background: The aim of this study was to analyse the effect of pomegranate juice (POM) supplementation on the levels of selected pro-inflammatory cytokines, hepcidin and markers of iron metabolism in well-trained rowers.

Method: The double-blind placebo-controlled study included 19 members of the Polish Rowing Team. The athletes were randomised into the supplemented group $(n=10)$, receiving $50 \mathrm{ml}$ of standardised POM daily for two months, or the placebo group $(n=9)$. The subjects performed a $2000 \mathrm{~m}$ test on the rowing ergometer at the start of the project (baseline) and end of follow-up period. Blood samples from the antecubital vein were obtained three times during each trial: prior to the exercise, one minute after the test, and following a $24 \mathrm{~h}$ recovery.

Results: The study documented the beneficial effect of supplementation with pomegranate fruit juice on TAC $(P<0.002)$. During the resting period, TAC level in the supplemented group was significantly higher than in the placebo group $(x \pm S D, 2.49 \pm 0.39$ vs. $1.88 \pm 0.45, P<0.05)$. The ergometric test conducted at baseline demonstrated a significant post-exercise increase in the concentrations of soluble transferrin receptors $(P<0.04)$, iron $(P<0.002)$ and IL-6 $(P<0.02)$, and to a significant post-exercise decrease in TAC. A significant increase in IL-6 concentration was also observed $24 \mathrm{~h}$ post-exercise. The exercise test conducted at the end of the follow-up period resulted in a significant decrease in TBIC and a significant increase in UIBC $(P<0.001)$, observed in both groups, both immediately post-exercise and after the resting period.
\end{abstract}

Conclusion: Supplementation with POM contributed to a significant strengthening of plasma antioxidant potential in the group of well-trained rowers, but had no effect on iron metabolism markers.

Keywords: Pomegranate, Strenuous exercise, Training, Inflammation

\section{Background}

Physical training, in particular strenuous exercises, places a considerable burden on an athlete's body. To achieve outstanding results in competitive sports, athletes need to bear large, and not infrequently extreme, training loads. This can result in a disruption to their intrinsic homeostasis, and thus have an unfavourable effect on their

\footnotetext{
* Correspondence: ankass@poczta.onet.pl

${ }^{1}$ Department of Morphological and Health Sciences, Faculty of Physical Culture in Gorzów Wlkp. Poland, 13 Estkowskiego Str., 66 - 400 Gorzów Wikp, Poland

Full list of author information is available at the end of the article
}

performance. Published evidence suggests that a key determinant of athletes' performance is iron metabolism $[1,2]$. Normal levels of iron are a prerequisite for many physiological processes, such as oxygen transport and energy synthesis [3].

The level of iron in the human body is affected not only by an adequate dietary intake of this element, but also by exercise-induced inflammation [4]. The discovery of hepcidin, a hormone, provided the link between iron deficiency and concomitant inflammation [5]. Studies have demonstrated that moderate-intensity training has a beneficial effect on iron metabolism, whereas strenuous 
exercise may induce systemic inflammation [6]. The inflammatory response is associated with enhanced synthesis of hepcidin; this hormone is involved in the degradation of ferroportin, and thus prevents the mobilisation of iron from its cellular deposits (e.g. in the liver and spleen) and interferes with its gastrointestinal absorption [7]. If this state persists, it may negatively affect erythrocyte parameters, and eventually lead to anaemia [8]. Reinke et al. [9] showed that at the end of competitive season, $70 \%$ of professional athletes (rowers and football players) presented with functional iron deficiency, and $27 \%$ had an absolute deficiency of this element. Unfavourable changes in iron parameters after strenuous exercise have also been reported by other authors [10].

Additional supplementation with iron-rich preparations does not necessarily bring expected results, and may be even harmful to an athlete's health $[11,12]$. In an animal study conducted by Reardon et al. [13], injection of iron contributed to an increase in muscle and plasma concentrations of this element and to exacerbation of oxidative stress; as a result, animals from iron-supplemented group presented with lower muscle power than the controls and performed less work during the treadmill test.

The post-exercise increase in hepcidin level has been also postulated to result from an increase in the so-called labile iron pool (LIP) [1], caused by enhanced haemolysis [14]. Products of heme degradation may stimulate the generation of reactive oxygen species, leading to further damage of erythrocyte membranes. The latter are abundant in polyunsaturated fatty acids, which makes them particularly prone to oxidative injury. This selfperpetuating process may last as long as its natural "attenuation" occurs.

Pomegranate juice (POM) from the fruits of Punnica Granatum L. is a rich source of polyphenols, such as anthocyanins, flavanols and some ellagitannins, especially punicalagin [15]. Many studies have documented the beneficial effects of POM consumption in the treatment of various disorders [16, 17]. Researchers have recently become increasingly interested in the dietary supplementation of athletes with POM. Fuster-Mũnoz et al. [18] demonstrated that POM exerted a positive effect on the modulation of fat and protein damage in well-trained endurance-based athletes. Ammar et al. [19] showed that the consumption of POM $48 \mathrm{~h}$ prior to, and during training sessions contributed to the alleviation of pain, delayed damage, inflammation, and soreness of the knee flexor, accelerated the recovery kinetics of biological parameters and improved performance in nine elite weightlifters.

One group of polyphenols in pomegranate juice are anthocyanins. These compounds have an array of biological activities, showing antioxidant properties [20], acting as immunostimulants, modulating inflammatory response [21] and chelating iron ions, which may contribute to the reduction of LIP [22].

We hypothesised that supplementation with pomegranate fruit juice may boost the antioxidant potential of the athletes, contributing to an increase in TAC, and may thus attenuate the inflammatory response triggered by intense physical exercise. We also examined whether, and to what extent, these changes affected iron metabolism parameters in the study subjects.

\section{Methods}

The protocol of the study was approved by the local bioethics committee at the University of Medical Sciences in Poznan (Decision no. 357/15). All athletes were adequately informed about the nature of the study and provided their written consent to participate in the project.

\section{Participants}

The study included a group of 19 male rowers, members of the Polish National Team, who participated in an eight-week training camp between the preparatory and competitive periods. The basic characteristics of the study athletes are shown in Table 1. The study subjects were randomised to one of two groups, receiving standardised POM (supplemented group, $n=10$ ) or a placebo (control group, $n=9$ ).

\section{Food intake}

Rowers from the supplemented group received $50 \mathrm{ml}$ of a standardised, commercially available POM (Oleofarm ${ }^{\circ}$ ) daily, for two months. The product was $100 \%$ pure natural juice squeezed from fresh fruit, with a total polyphenol content equal to $220 \mathrm{mg} / 100 \mathrm{~g}$. Athletes from the non-supplemented group received the same dose of a placebo composed of water, sugar and grenadine, with a colour and taste resembling that of the pomegranate fruit juice. Both pomegranate fruit juice and placebo were provided by the same manufacturer $\left(\right.$ Oleofarm ${ }^{\circ}$ ), packed in identical dark bottles labelled with encoded information about the type of preparation and its recommended dosage. The labels were decoded at the end of the study.

The athletes completed food intake questionnaires on each day of the study period, which were later used to

Table 1 Basic characteristics of the study groups (mean \pm standard deviation)

\begin{tabular}{lll}
\hline Parameter & $\begin{array}{l}\text { Supplemented group } \\
(n=10)\end{array}$ & $\begin{array}{l}\text { Control group } \\
(n=9)\end{array}$ \\
\hline Age (years) & $20.8 \pm 0.86$ & $20.9 \pm 0.95$ \\
Body weight $(\mathrm{kg})$ & $89.4 \pm 8.97$ & $83.85 \pm 12.04$ \\
Body height $(\mathrm{cm})$ & $192.1 \pm 6.64$ & $189.6 \pm 5.79$ \\
Duration of training (years) & $8.2 \pm 0.78$ & $7.14 \pm 0.69$ \\
\hline
\end{tabular}


calculate the energy equivalents for their diet and the dietary intake of antioxidants and vitamins. All study subjects agreed that they refrained from drugs, medications and dietary supplements for at least two weeks preceding the study and throughout the whole study period.

\section{Experimental procedure}

The characteristics of training profiles, such as intensity, volume (in $\mathrm{min}$ ) and type (specific, i.e. rowing: endurance, technical, speed, etc., and non-specific: jogging, strength) were recorded on a daily basis. Training intensity was classified in relation to the lactic acid (LA) threshold $(4 \mathrm{mmol} / \mathrm{L})$, as an extensive (below the LA threshold) or intensive (above the LA threshold) workload (Table 2). On the first day (prior to supplementation, at the baseline) and at the end of the training camp (after supplementation, at the end of the follow-up period), the athletes performed a controlled 2000-m rowing exercise test (Concept II, Model D, USA). All study subjects were asked to perform both tests at their maximal pace. Each test was preceded by a five minute warm-up session.

Blood samples were collected for the analysis $(0.9 \mathrm{ml})$ prior to each $2000 \mathrm{~m}$ test (in the morning, after an overnight fasting), one minute after the test and following a $24 \mathrm{~h}$ recovery period. Immediately after collection, the samples were centrifuged to separate erythrocytes from serum. The serum was immediately frozen and stored at $-80{ }^{\circ} \mathrm{C}$ until the analysis. Capillary blood samples were obtained via an ear lobe prick before and after each exercise test, to assess LA levels.

\section{Measurements}

Total antioxidant capacity (TAC), was measured as an indicator of plasma antioxidant capacity with a commercially available ELISA kit (Cayman, cat no. Antioxidant Assay 709,001-96, USA); the results were expressed in $\mathrm{mmol} / \mathrm{L}$. Uric acid (UA) level was determined with a commercially available kit (Alpha Diagnostics, Cat No. K6681-100); the results were expressed in $\mathrm{mg} / \mathrm{dL}$. Serum interleukin 6 (IL-6) was quantified with a commercially available enzyme-linked immunosorbent assay (ELISA; Quantikine HS, R\&D Systems, Minneapolis, USA); the results were expressed in $\mathrm{pg} / \mathrm{ml}$. Serum hepcidin was measured using a commercially available ELISA kit (Wuhan EIAab Science Co., China); the results were expressed in $\mathrm{ng} / \mathrm{mL}$. Iron concentration and TIBC were determined using colorimetric method with chromogens (cat. no. 1-418-01-50; 1-421-0060 BioMaxima, Poland); the results were expressed in $\mu \mathrm{g} / \mathrm{dL}$. Unsaturated ironbinding capacity (UIBC) was calculated from the formula: $\mathrm{UIBC}=$ TIBC - Fe. Myoglobin concentration was determined immunochemically, using Myoglobin ELISA kit (Biocom, cat. no. 11170); the results were expressed in $\mathrm{ng} / \mathrm{mL}$. Serum ferritin was quantified immunochemically, with a commercially available diagnostic kit (Demeditec, Germany); the results were expressed in $\mathrm{ng} / \mathrm{ml}$. Concentration of soluble transferrin receptor (sTfR) was determined immunochemically, with a commercially available diagnostic kit (Biocom, cat. no. RD194011100); the results were expressed in $\mu \mathrm{g} / \mathrm{mL}$. Creatine kinase (CK) activity in blood plasma was determined with a commercially available kit (Dr Lange, Germany, cat. no. LCN 282); the results were expressed in U/L. The coefficients of variation for all assays were $<12 \%$.

Table 2 Training schedules during the weeks preceding blood samples before (Trial I) and after (Trial II) the supplementation

\begin{tabular}{|c|c|c|c|c|c|c|c|}
\hline & \multicolumn{7}{|c|}{ Days before blood sample collection } \\
\hline & 1 & 2 & 3 & 4 & 5 & 6 & 7 \\
\hline Total training time [min/day] & 120 & 100 & 200 & 190 & 210 & 150 & 120 \\
\hline Time rowed [min/day] & 110 & 100 & 100 & 100 & 70 & 90 & 100 \\
\hline Distance rowed [km/day] & 22 & 20 & 20 & 20 & 16 & 18 & 20 \\
\hline Training for force development [min/day] & - & - & 90 & - & 70 & - & - \\
\hline Extensive endurance rowing training time [min/day] & 70 & 100 & 100 & 40 & 40 & 90 & 100 \\
\hline High-intensity endurance rowing training time [min/day] & 40 & - & - & 60 & 30 & - & - \\
\hline Unspecific training (running etc.) [min/day] & 10 & - & 10 & 90 & 70 & 60 & 20 \\
\hline Total training time [min/day] & 180 & 100 & 190 & 160 & 200 & 90 & 130 \\
\hline Time rowed [min/day] & 160 & 100 & 130 & 140 & 120 & 90 & 125 \\
\hline Distance rowed [km/day] & 32 & 18 & 26 & 28 & 20 & 16 & 20 \\
\hline Training for force development [min/day] & - & - & 60 & - & 60 & - & - \\
\hline Extensive endurance rowing training time [min/day] & 160 & 100 & 130 & 90 & 94 & 90 & 125 \\
\hline High-intensity endurance rowing training time [min/day] & - & - & - & 30 & 26 & - & 5 \\
\hline Unspecific training (running etc.) [min/day] & 20 & - & 25 & 20 & 20 & - & - \\
\hline
\end{tabular}


The concentration of LA in capillary blood was measured immediately after sampling, with a commercially available kit (cat. no. LKM 140, HACH LANGE, Düsseldorf, Germany); the results were presented in $\mathrm{mmol} / \mathrm{L}$.

\section{Statistical analysis}

Statistical analysis of the results was carried out with the STATISTICA v. 10.0 software package (StatSoft, Cracow, Poland). The significance of intergroup and intragroup differences was verified using 2 (supplemented and placebo group) $\times 3$ (timing of measurement) repeated measures analysis of variance (ANOVA). Normal distribution of the study variables was verified with a Shapiro-Wilk test. When statistically significant differences were documented on ANOVA, Fisher's post-hoc tests were conducted to identify the source of variance. The anthropometric characteristics of the study groups were compared with unpaired Student $t$-tests. Except for the rowing time, the results of the $2000 \mathrm{~m}$ tests performed prior to and after the supplementation period were subjected to intragroup and intergroup comparisons with paired and unpaired Student $t$-tests, respectively. The results of the $2000 \mathrm{~m}$ simulated rowing test were subjected to one-way ANOVA. The statistical characteristics of the study variables are presented as arithmetic means \pm standard deviations (SD). The threshold of statistical significance for all tests was set at $p<0.05$.

\section{Results}

Athletes from the supplemented group did not differ significantly from the controls in terms of their anthropometric parameters, age and training experience (Table 1). No statistically significant intergroup differences were found in power output, total row time over a $2000 \mathrm{~m}$ distance, or pre- and post-test LA levels (Table 3). The TAC values were determined prior to and after the supplementation period, and are presented in Fig. 1a. TAC turned out to be modulated by both physical exercise $(p<0.029)$ and POM supplementation $(p<0.002)$. In the baseline measurements, athletes from both study groups showed a post-exercise decrease in TAC levels. At the end of the follow-up, post-recovery TAC level in athletes from the supplemented group was significantly higher than in the controls. Regardless of the study group, post-recovery UA concentrations at baseline were significantly higher $(p<0.0004)$ than those determined immediately after the exercise test (Fig. 1b). Supplementation with POM had no significant effects on serum hepcidin, myoglobin or creatine kinase levels (Fig. 2a, b, d). Athletes from both groups showed a significant post-exercise increase in iron level $(p<0.002$, Fig. 2c) at baseline measurements. Statistically significant changes in UIBC and TIBC were observed in both study groups, but were statistically significant post-intervention (Fig. 3a and b respectively). UIBC increased immediately after the exercise test $(p<0.001)$ and remained elevated until the end of the recovery period. The exercise test contributed to a significant decrease in TIBC level $(\mathrm{p}<0.001)$, which persisted at the end of the recovery period. ANOVA did not demonstrate the significant effect of POM supplementation on any of these parameters. None of the study groups showed significant changes in ferritin levels at any point of the study (Fig. 3c). The results for sTfR are presented in Fig. 3d. A significant post-exercise increase in sTfR was observed at baseline in the supplemented group $(p<0.04)$, along with a significant decrease in this parameter in the controls. Post-intervention, athletes from the supplemented group presented with significantly higher pre-exercise levels of sTfR than the controls $(p<0.012)$. Pre- and post-supplementation changes in IL- 6 levels are shown in Fig. 4. As demonstrated in the ANOVA, physical exercise had a significant effect on the concentration of this cytokine $(p<0.02)$. Prior to the supplementation period, athletes from both groups showed a significant post-exercise increase in IL-6 levels, which persisted post-recovery. This effect was no longer observed at the end of the follow-up period.

\section{Discussion}

In this study, supplementation with pomegranate fruit juice boosted the antioxidant potential of rowers, as expressed by TAC. The level of this parameter in the supplemented group was significantly higher during the restitution period than in the placebo group (Fig. 1a).

Table 3 Changes in a 2000 m rowing ergometer performance before and after the supplementation

\begin{tabular}{|c|c|c|c|c|}
\hline \multirow[t]{2}{*}{ Parameters } & \multicolumn{2}{|c|}{ Supplemented group $(n=10)$} & \multicolumn{2}{|c|}{ Control group $(n=9)$} \\
\hline & Before & After & Before & After \\
\hline Power (watt) & $432 \pm 38.4$ & $439 \pm 36.4$ & $424 \pm 41.3$ & $430 \pm 45.6$ \\
\hline (W/kg) & $4.92 \pm 0.29$ & $4.93 \pm 0.33$ & $4.96 \pm 0.24$ & $5.03 \pm 0.20$ \\
\hline$L A_{\min }(\mathrm{mmol} / \mathrm{L})^{\mathrm{a}}$ & $1.8 \pm 0.48$ & $1.5 \pm 0.26$ & $1.8 \pm 0.25$ & $1.6 \pm 0.23$ \\
\hline$L \mathrm{~A}_{\max }(\mathrm{mmol} / \mathrm{L})^{\mathrm{a}}$ & $15.2 \pm 2.72$ & $16.33 \pm 4.11$ & $13.0 \pm 1.84$ & $14.44 \pm 2.97$ \\
\hline Time $(s)$ & $373 \pm 11.5$ & $371 \pm 10.3$ & $375 \pm 12.02$ & $378 \pm 15.56$ \\
\hline
\end{tabular}

Values represent mean \pm standard deviation. No significant differences were found between pre- and post-supplementation parameters $(p<0.05)$

aA lactic acid 


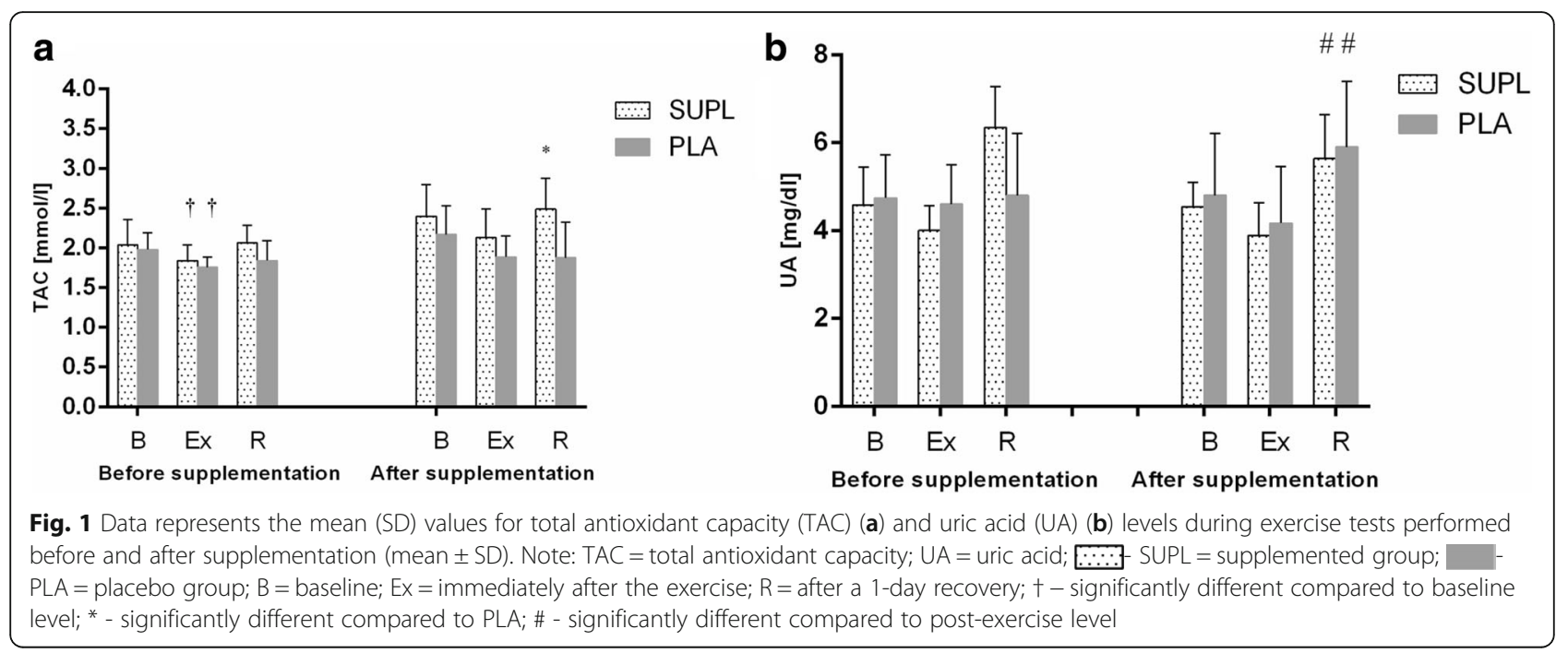

The increase in antioxidant potential did not exert a significant effect on other study parameters, however. Previous studies [23] have demonstrated that pomegranate fruit juice has three-fold greater antioxidant activity than other food products widely recognised for their antioxidant properties, such as red wine and green tea. The antioxidant potential of pomegranate fruit juice results from its high content of polyphenols, especially proanthocyanidins [24]. An increase in TAC after a two week supplementation with pomegranate fruit juice has also been reported by other authors [25].
Prior to the supplementation (at baseline), intense physical exercise resulted in a significant decrease in TAC in the study athletes (Fig. 1). Free radicals that are accumulated in excess and inadequately inactivated may, inter alia, initiate the peroxidation of polyunsaturated fatty acids of erythrocyte membranes, and thus enhance post-exercise haemolysis [26, 27]. This hypothesis might also be supported by the observation that prior to supplementation, our rowers showed greater post-exercise increases in iron concentration (Fig. 2c). Manthou et al. [28] demonstrated that healthy subjects supplemented

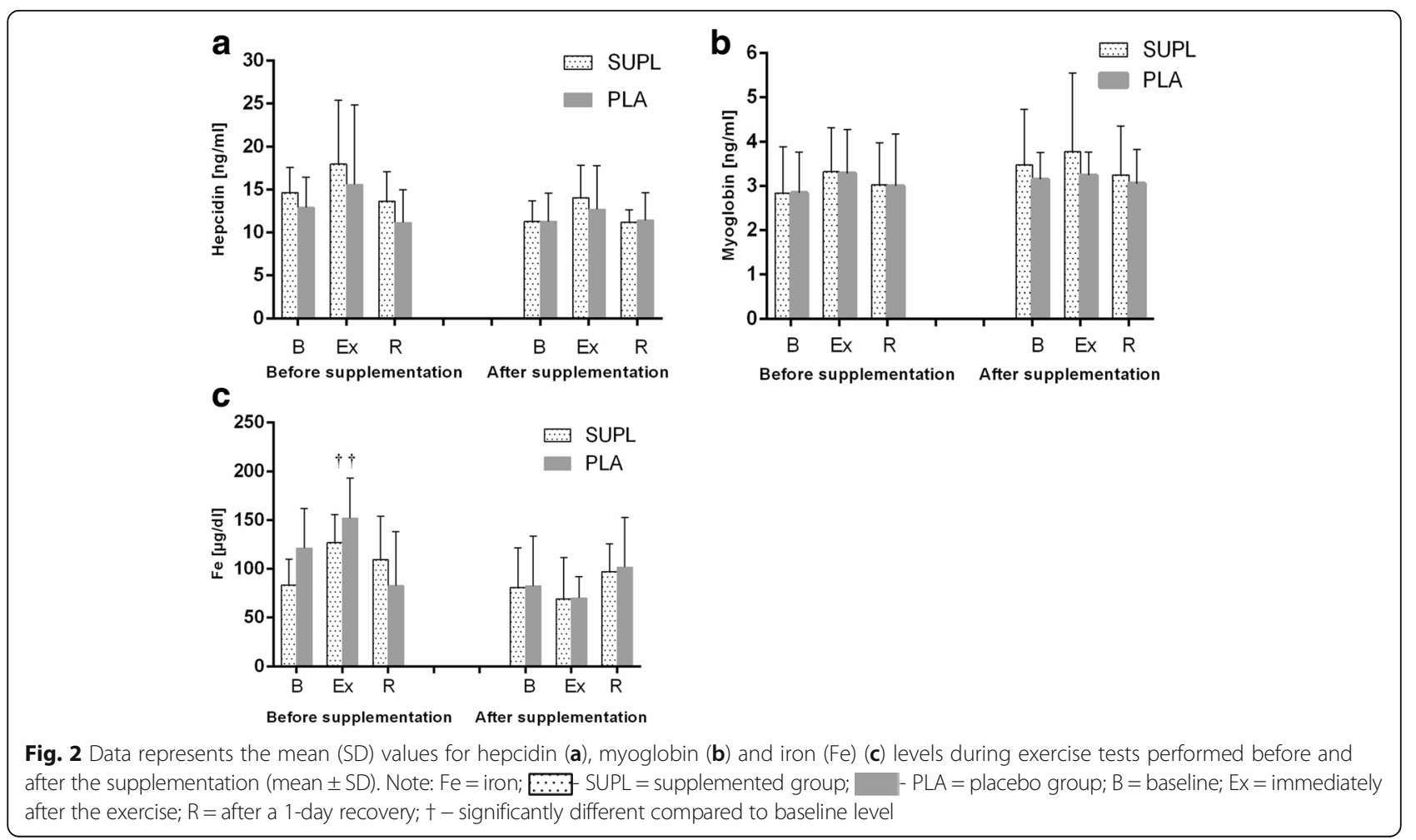




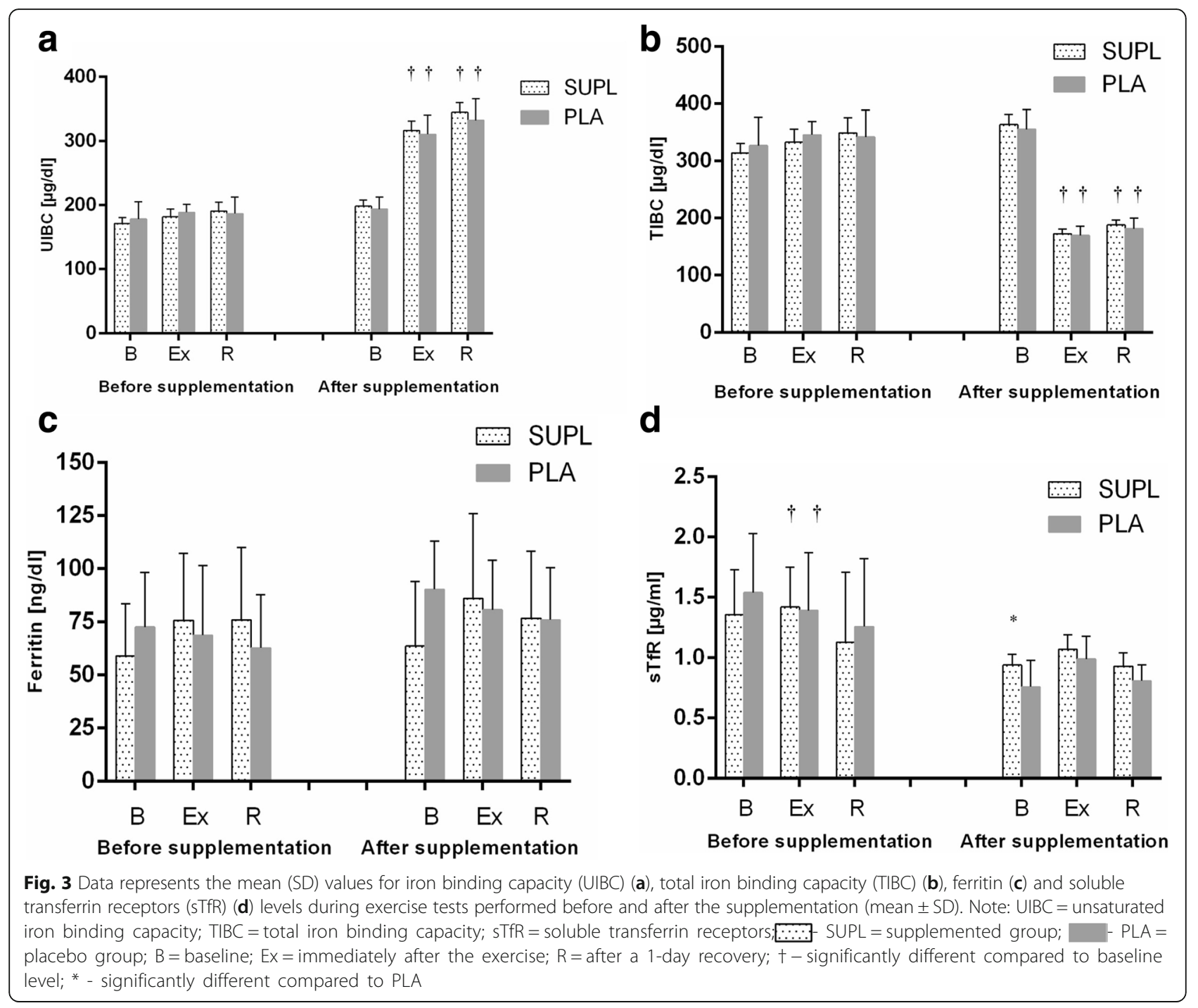

for 14 days with pomegranate fruit juice had increased RBC count, haemoglobin concentration and haematocrit levels. According to those authors, these favourable changes might result from more effective prevention of RBC degradation among other thinks. Fiorani et al. [29] demonstrated that human erythrocytes can absorb extracellular flavonoids via passive diffusion, and constitute a reservoir of these compounds. While most flavonoids (according to the authors, up to 85\%) reach the cytosol, some are incorporated into cell membrane. Studies $[30,31]$ have shown that, similar to cholesterol and alpha-tocopherol, intracellular flavonoids are localised in close proximity to the cell membrane, between the lipid bilayer and aqueous phase. As a result of this location, flavonoids play a vital role in the cell, stabilising plasma membranes that become less fluid, and thus, more resistant to oxidation [32]. Another key issue is cooperation between flavonoids, alpha-tocopherol and ascorbic acid. Flavonoids were shown to inhibit the oxidation of intracellular alpha-tocopherol and to regenerate (as does vitamin C) oxidised alpha-tocopherol to its radical. Ascorbic acid, also protected by flavonoids against oxidation, can in turn inhibit oxidative changes in flavonoids, prolonging their protective effect $[33,34]$. Flavonoids therefore maintain a relative balance between oxidised and reduced forms of antioxidants and their radicals, and therefore provide another protective mechanism against elevated concentrations of reactive oxygen species.

Although only athletes from the supplemented group presented with enhanced antioxidant potential during the ergometric test conducted at the end of the follow-up period, physical exercise did not induce significant changes in TAC in either study group (Fig. 1a). Uric acid, the final product of purine metabolism, which proved to be an important antioxidant of blood plasma during in vivo studies [35], did not contribute to changes in TAC levels, although our rowers presented with elevated 


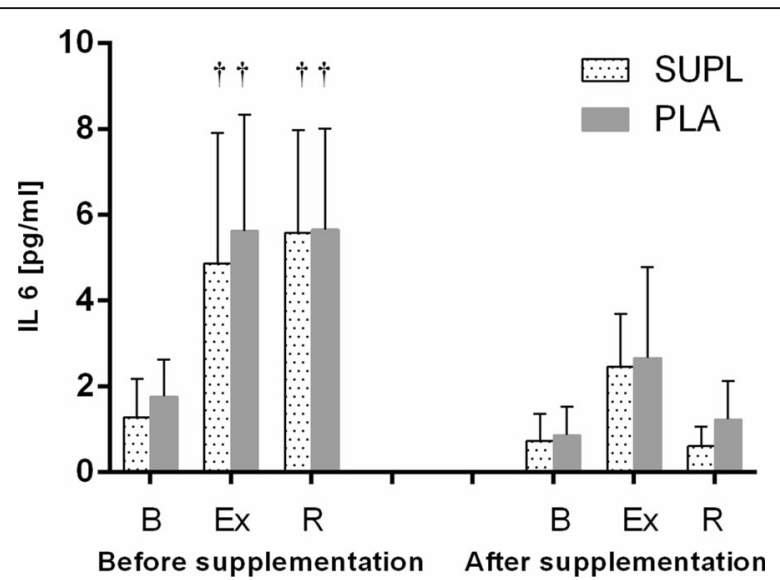

Fig. 4 Data represents the mean (SD) values for interleukin 6 (IL-6) levels during exercise tests performed before and after the supplementation (mean \pm SD). Note: IL $6=$ interleukin $6 ; \ldots$ SUPL = supplemented group; $\quad$ - PLA = placebo group; $B=$ baseline; $E x=$ immediately after the exercise; $\mathrm{R}=$ after a 1-day recovery; $†-$ significantly different compared to baseline level

concentrations during the restitution period (Fig. 1b). Braakhuis et al. [36] demonstrated that the result of a $30 \mathrm{~min}$ rowing-ergometer test correlated positively with years and hours of training and the antioxidant status of the blood in elite rowers. According to those authors, these factors had a greater impact on TAC than the dietary intake of antioxidants. The results of our present study suggest that another modulator of TAC may be the phase of the training cycle. The second ergometric test took place during the competitive period when the organism of a well-trained athlete should be characterised by so-called "readiness for competition", that is be fully adapted to an exercise load specific for a given discipline. It should be stressed that during rowing competitions, athletes participate in qualification and final races, and sometimes need to cover a $2000 \mathrm{~m}$ distance twice in a single day. The adaptation of our rowers to this type of exercise load was confirmed by other parameters analysed: a lack of statistically significant changes in IL-6 concentration (Fig. 4) and post-exercise increases in iron levels (Fig. 2c). A study of elite male rowers conducted prior to the Rowing World Championships showed a significant association between the level of proinflammatory cytokines, such as IL-1 $\beta$, TNF- $\alpha$ and IL- 6 , and measures of depressed mood, sleep disturbances and fatigue [37]. The lack of statistically significant post-exercise changes in concentrations of proinflammatory cytokines may thus provide important information about the readiness of athletes for competition.

Irrespective of the testing period, our athletes did not show statistically significant changes in hepcidin, myoglobin or CK levels (Fig. 2a, b, Fig. 5). To the best of our knowledge, CK activity has rarely been studied

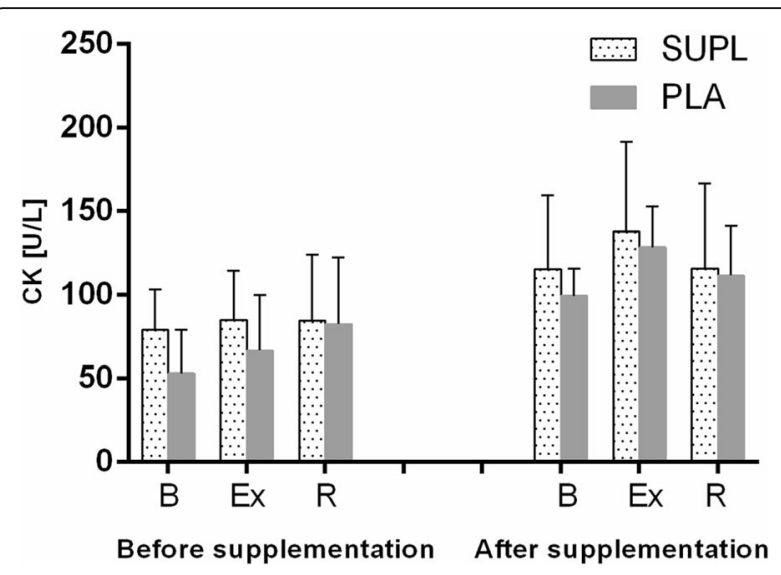

Fig. 5 Data represents the mean (SD) values for creatine kinase (CK) levels during exercise tests performed before and after the supplementation (mean \pm SD). Note: $C K=$ creatine kinase; $\ldots .$. SUPL = supplemented group; _ - PLA = placebo group; $B=$ baseline; Ex $=$ immediately after the exercise; $R=$ after a 1 - day recovery

in POM-supplemented subjects. We found only one report documenting a significant increase in CK activity in a group of recreationally active males receiving either POM or a placebo for a period of nine days; this effect was probably a consequence of myocyte damage in both study groups [38]. The lack of significant changes in hepcidin, myoglobin and CK levels in our study subjects could perhaps be explained by their good adaptation to large training loads; this issue seems to be an interesting topic for future research. Nevertheless, athletes from both groups showed a significant post-exercise increase in serum concentration iron at baseline measurements (Fig. 2c).

Both supplemented athletes and controls showed a significant post-exercise increase in UIBC during the follow-up test, which persisted after a $24 \mathrm{~h}$ recovery (Fig. 3a). Similarly, the post-exercise changes in TIBC seemed to be supplementation-independent, since a significant post-exercise decrease in this parameter was observed post-intervention regardless of the study group, both immediately after the ergometric test and following a $24 \mathrm{~h}$ recovery (Fig. 3b). Monitoring of sTfR and body iron has previously shown to be a reliable tool for the determination of Fe metabolism and successful prevention of its deficiency [39]. In our present study, ergometric tests conducted at the baseline contributed to a significant increase in sTfR level in the supplemented group and to a significant decrease in this parameter in the controls. Noticeably, athletes from the supplemented group presented with significantly higher pre-exercise levels of sTfR than the controls during the post-intervention ergometric test (Fig. 3d). Neither supplementation with POM nor physical exercise had a significant effect on serum ferritin levels in our study 
subjects (Fig. 3c), which is consistent with the results of other studies $[40,41]$.

\section{Conclusions}

This study showed that the administration of pomegranate fruit juice, a dietary supplement with established high antioxidant potential, boosted the TAC of the study athletes, but had no significant effect on inflammatory markers or other parameters analysed. In the case of well-trained athletes, the training phase and adaptation to exercise loads also seem to be important determinants, but this hypothesis needs to be verified by further comprehensive studies.

\section{Abbreviations}

ANOVA: Analysis of variance; Fe: Serum iron; IL - 1ß: Interleukin 1 beta; IL6: Interleukin; LA: Lactic acid; LIP: Labile iron pool; POM: Pomegranate juice; SD: Standard deviations; sTfR: Soluble transferrin receptor; TAC: Total antioxidant capacity; TIBC: Total iron binding capacity; TNF-a: Tumour necrosis factor alpha; UIBC: Unsaturated iron-binding capacity

\section{Acknowledgements}

The authors would like to thank the Fund for the Development of Young Scientists for financial support for AU.

\section{Availability of data and materials}

The datasets used and/or analysed during the current study are available from the corresponding author for a reasonable request.

\section{Author contributions}

ASS, AU and KA designed the study; PB, AW, KWJ and KA collected the data, $A U, E L$ and ASS interpreted the results and drafted the manuscript. All authors approved the final version of the paper.

\section{Funding}

This work was supported by the Polish Ministry of Science and Higher Education -Development of Young Scientists.

\section{Ethics approval and consent to participate}

Experimental procedures and potential risks were discussed with the participants, and informed consent forms were provided and signed prior to inclusion in the study. The study was conducted in accordance with the Declaration of Helsinki, and its protocol was approved by the Local Ethics Committee at Poznan University of Medical Sciences (Decision no. 769/13 of 10 October 2013).

\section{Consent for publication}

Not applicable.

\section{Competing interests}

The authors declare no conflict of interest, financial or otherwise. The authors declare that the results of the study are presented clearly, honestly, and without fabrication, falsification or inappropriate data manipulation.

\section{Publisher's Note}

Springer Nature remains neutral with regard to jurisdictional claims in published maps and institutional affiliations.

\footnotetext{
Author details

${ }^{1}$ Department of Morphological and Health Sciences, Faculty of Physical Culture in Gorzów Wlkp. Poland, 13 Estkowskiego Str., 66 - 400 Gorzów Wlkp, Poland. ${ }^{2}$ Department of Water Sports, Faculty of Physical Culture in Gorzów Wlkp. Poland, 13 Estkowskiego Str., 66 - 400 Gorzów WIkp, Poland. ${ }^{3}$ Jacob of Paradies University in Gorzów Wielkopolski, Teatralna Str. 25, Gorzów Wielkopolski 66-400, Poland. ${ }^{4}$ Faculty of Physical Culture in Gorzów Wlkp, 13 Estkowskiego Str., 66 - 400 Gorzów Wlkp, Poland.
}

Received: 19 March 2018 Accepted: 16 July 2018

Published online: 24 July 2018

\section{References}

1. Buratti P, Gammella E, Rybinska I, Cairo G, Recalcati S. Recent advances in iron metabolism: relevance for health, exercise, and performance. Med Sci Sports Exerc. 2015;47(8):1596-604

2. Otto J, Montgomery $\mathrm{H}$, Richards T. Haemoglobin concentration and mass as determinants of exercise performance and of surgical outcome. Extrem Physiol Med. 2013;2(1):33.

3. Gozzelino R, Arosio P. Iron homeostasis in health and disease. Int J Mol Sci. 2016;17(1):1-14

4. Dahlquist DT, Stellingwerff T, Dieter BP, McKenzie DC, Koehle MS. Effects of macro- and micronutrients on exercise-induced hepcidin response in highly trained endurance athletes. Appl Physiol Nutr Metab. 2017:42(10):1036-43.

5. Nemeth E, Tuttle MS, Powelson J, Vaughn MB, Donovan A, Ward DM et al. Hepcidin regulates cellular iron efflux by binding to ferroportin and inducing its internalization. Science 2004; 306(5704):2090-2093.

6. Kong W, Gao G, Chang Y. Hepcidin and sports anemia. Cell Biosc 2014;419. https://doi.org/10.1186/2045-3701-4-19.

7. Sukumaran A, Varghese J, Tamilselvan J, Jeyaseelan V, Mani T, Simpson RJ, et al. Effects of acute and chronic inflammation on proteins involved in duodenal iron absorption in mice: a time-course study. Bri Journal Nutri 2012:108(11):1994-2001.

8. Yu-Qian L, Yan-Zhong C, Bin Z, Hai-Tao W, Xiang-Lin D. Does hepatic hepcidin play an important role in exercise-associated anemia in rats? Int J Sport Nutr Exerc Metabol. 2011;21(1):19-26.

9. Reinke S, Taylor WR, Duda GN, von Haehling S, Reinke P, Volk H. et al. Absolute and functional iron deficiency in professional athletes during training and recovery. Internat J Cardiol 2012; 156(2):186-191.

10. Merkel D, Huerta M, Grotto I, Blum D, Tal O, Rachmilewitz E. et al. Prevalence of iron deficiency and anemia among strenuously trained adolescents. J Adolesc Health 2005;37(3):220-223.

11. Clénin GE, Cordes M, Huber A, Schumacher YO, Noack P, Scales J. et al. Iron deficiency in sports - definition, influence on performance and therapy Swiss Med Wkly 2016;64(1):6-18.

12. Ishibashi A, Maeda N, Kamei A, Goto K. Iron supplementation during three consecutive days of endurance training augmented hepcidin levels. Nutrients. 2017;9(8). https://doi.org/10.3390/nu9080820.

13. Reardon TF, Allen DG. Iron injections in mice increase skeletal muscle iron content, induce oxidative stress and reduce exercise performance. Exp Physiol. 2009:94(6):720-30.

14. Berzosa C, Gómez-Trullén EM, Piedrafita E, Cebrián I, Martínez-Ballarín E, Miana-Mena FJ, et al. Erythrocyte membrane fluidity and indices of plasmatic oxidative damage after acute physical exercise in humans. Eur J Appl Physiol 2011;111(6):1127-1133.

15. Gil M, Tomás-Barberán F, Hess-Pierce B, Holcroft DM, et al. Antioxidant activity of pomegranate juice and its relationship with phenolic composition and processing. J Agric Food Chem 2000;48: 4581-4589.

16. Faria A, Monteiro R, Mateus N, Azeveda I, Calhau C. Effect of pomegranate (Punica granatum) juice intake on hepatic oxidative stress. Eur J Nutr. 2007:46:271-8.

17. Shema-Didi L, Sela S, Ore L, Shapiro G, Geron R, Moshe G, Kristal B. One year of pomegranate juice intake decreases oxidative stress, inflammation, and incidence of infections in hemodialysis patients: a randomised placebo controlled trial. Free Radic Biol Med. 2012:53:297-304.

18. Fuster-Mũnoz E, Roche E, Funes L, Martinez-Peinado P, Sempere JM, Vicente-Salar N. Effects of pomegranate juice in circulating parameters, cytokines, and oxidative stress markers in endurance-based athletes: a randomised controlled trial. Nutrition. 2016:32:539-45.

19. Ammar A, Turki M, Chtourou H, Hammouda O, Trabelsi K, Kallel C, et al. Pomegranate supplementation accelerates recovery of muscle damage and soreness and inflammatory markers after a weightlifting training session. PLoS One, 2016:1-19.

20. Căta A, Ştefănuț MN, Pop R, Tănasie C, Moşoarcă C, Zamfir AD, et al. Evaluation of antioxidant activities of some small fruits containing anthocyanins using electrochemical and chemical methods. Croat Chem Acta 2016; 89(1):37-48

21. Karlsen A, Retterstøl L, Laake P, Paur I, Kjølsrud-Bøhn S, Sandvik L, et al. Anthocyanins inhibit nuclear factor-kb activation in monocytes and reduce plasma concentrations of pro-inflammatory mediators in healthy adults. J Nutr 2007;137(8):1951-1954. 
22. Kelsey N, Hulick W, Winter A, Ross E, Linseman D. Neuroprotective effects of anthocyanins on apoptosis induced by mitochondrial oxidative stress. Nutr Neurosc. 2011;14(6):249-59.

23. Gil M, Holcroft D, Kader A, Tomas-Barberan A, Hess-Pierce B. Antioxidant activity of pomegranate juice and its relationship with phenolic composition and processing. J Agric Food Chem. 2000;48(10):4581-9.

24. El Kar C, Ferchichi A, Attia F, Bouajila J. Pomegranate (Punica granatum) juices: chemical composition, micronutrient cations, and antioxidant capacity. J Food Sci. 2011;76(6):C795-800.

25. Naghizadeh-Baghi A, Mazani M, Shadman-Fard A, Nemati A. Punica granatum juice effects on oxidative stress in severe physical activity. Mater Sociomed. 2015;27(1):48-51.

26. Beneke R, Bihn D, Hütler M, Leithäuser R. Haemolysis caused by alterations of $\alpha$ - and $\beta$-spectrin after 10 to 35 min of severe exercise. Eur J Appl Physiol. 2005:95(4):307-12.

27. Bonilla JF, Narváez R, Chuaire L. Sports as a cause of oxidative stress and hemolysis. Colombia Medica. 2005;36(4):281-6

28. Manthou E, Gergakouli K, Deli CK, Sotiropoulos A, Fatouros IG, Kouretas D, et al. Effect of pomegranate juice consumption on biochemical parameters and complete blood count. Exp Therap Med 2017;14:1756-1762.

29. Fiorani M, Accorsi A, Cantoni O. Human red blood cells as a natural flavonoid reservoir. Free Radic Res. 2003:37(12):1331-8.

30. Arora A, Byrem TM, Nair MG, Strasburg GM. Modulation of liposomal membrane fluidity by flavonoids and isoflavonoids. Arch Biochem Biophys. 2000;373(1):102-9.

31. Terao J, Piskula M, Yao Q. (1994). Protective effect of epicatechin, epicatechin gallate, and quercetin on lipid peroxidation in phospholipid bilayers. Arch Biochem Biophys. 1994;308(1):278-84.

32. Cherubini $A$, Beal MF, Frei B. Black tea increases the resistance of human plasma to lipid peroxidation in vitro, but not ex vivo. Free Radic Biol Med. 1999;27(3-4):381-7.

33. Block G, Henson D, Levine M. Vitamin c: a new look. Ann Intern Med. 1991; 114(10):909-12.

34. Clemetson CA, Andersen L. Plant polyphenols as antioxidants for ascorbic acid. Ann N Y Acad Sci. 1966:136(14):341-76.

35. Kaur $\mathrm{H}$, Halliwell B. Action of biologically-relevant oxidizing species upon uric acid. Identification of uric acid oxidation products. Chem Biol Interact. 1990;73(2-3):235-47.

36. Braakhuis AJ, Hopkins WG, Lowe TE. Effect of dietary antioxidants, training, and performance correlates on antioxidant status in competitive rowers. Int J Sports Physiol Perform. 2013;8:565-72.

37. Main LC, Dawson B, Heel K, Grove JR, Landers GJ, Goodman C. Relationship between inflammatory cytokines and self-report measures of training overload. Res Sport Med. 2010;18(2):127-39.

38. Trombold JR, Reinfeld AS, Casler JR, Coyle EF. The effect of pomegranate juice supplementation on strength and soreness after eccentric exercise. J Strength Cond Res. 2011;25(7):1782-8.

39. Milic R, Martinovic J, Dopsaj M, Dopsaj V. Haematological and iron-related parameters in male and female athletes according to different metabolic energy demands. Eur J Appl Physiol. 2011;11::449-58.

40. Badenhorst C, Dawson B, Cox G, Laarakkers C, Swinkels D, Peeling P, et al. Acute dietary carbohydrate manipulation and the subsequent inflammatory and hepcidin responses to exercise. Eur J Appl Physiol 2015; 115(12), 2521-2530.

41. Burden RJ, Pollock N, Whyte GP, Richards T, Moore B, Busbridge M, et al. Effect of intravenous iron on aerobic capacity and iron metabolism in elite athletes. Med Sci Sports Exerc 2015; 47(7), 1399-1407.

Ready to submit your research? Choose BMC and benefit from:

- fast, convenient online submission

- thorough peer review by experienced researchers in your field

- rapid publication on acceptance

- support for research data, including large and complex data types

- gold Open Access which fosters wider collaboration and increased citations

- maximum visibility for your research: over $100 \mathrm{M}$ website views per year

At BMC, research is always in progress.

Learn more biomedcentral.com/submissions 